\title{
Assessing Regularity and Variability of Cortical Folding Patterns of Working Memory ROIs
}

\author{
Hanbo Chen ${ }^{1}$, Tuo Zhang ${ }^{2}$, Kaiming $\mathrm{Li}^{2}$, Xintao $\mathrm{Hu}^{2}$, Lei Guo ${ }^{2}$, and Tianming $\mathrm{Liu}^{1}$ \\ ${ }^{1}$ Department of Computer Science and Bioimaging Research Center, \\ The University of Georgia, Athens, GA, USA \\ ${ }^{2}$ School of Automation, Northwestern Polytechnical University, Xi' an, China
}

\begin{abstract}
Cortical folding patterns are believed to be good predictors of brain cytoarchitecture and function. For instance, neuroscientists frequently apply their domain knowledge to identify brain Regions of Interests (ROIs) based on cortical folding patterns. However, quantitative mapping of cortical folding pattern and brain function has not been established yet in the literature. This paper presents our initial effort in quantification of the regularity and variability of cortical folding pattern features for working memory ROIs identified by taskbased fMRI, which is widely accepted as a standard approach to localize functionally-specialized brain regions. Specifically, we used a set of shape attributes for each ROI base on multiple resolution decomposition of cortical surfaces, and described the meso-scale folding pattern via a polynomial-based approach. We also applied brain atlas label distribution as a global-scale description of ROI folding pattern. Our studies suggest that there is deep-rooted regularity of cortical folding patterns for certain working memory ROIs across subjects, and folding pattern attributes could be useful for the characterization, recognition and prediction of ROIs, if extracted and applied in a proper way.
\end{abstract}

Keywords: ROI, folding pattern, prediction.

\section{Introduction}

The relationship between brain anatomy and its function has been of keen interest for years. Extensive neuroscience research suggests that the cortical folding process and axongenesis process are closely coupled [1]. Since the structural connectivity pattern has been shown to be good predictor of function [2], it is reasonable to believe that cortical folding is a good predictor of brain function as well. For instance, the cortical folding patterns are shown to be good predictors of the brain cytoarchitecture [3]. However, quantitative mapping of cortical folding pattern and brain function has not been adequately studied yet in the literature. The accomplishment of such a goal entails the availability of both quantitative cortical folding pattern descriptors and accurate localizations of functionally-specialized brain regions.

This paper presents our initial effort in quantitative mapping of the relationship between cortical folding patterns and brain function, particularly in quantification of the regularity and variability of folding pattern features for working memory ROIs. First, we identify brain regions involved in the working memory system by using 
task-based fMRI. Then, we introduce two groups of descriptive cortical folding pattern features for these ROIs in multiple scales. One is to present the global-scale folding pattern by using the widely used MNI (Montreal Neurological Institute) atlas labels. Another is to present the meso-scale folding pattern by using the recently published polynomial models [4]. Compared with other folding pattern descriptors [5, $6,7]$, the advantages of the polynomial model not only lie in its meso-scale view which is more suitable for ROIs, but also its compactness for describing the symmetric shape patterns and its soundness for classifying the cortical shapes into eight primitive folding patterns [8]. To effectively extract folding pattern features for the ROIs, the cortical surface is decomposed into multiple resolutions [7] and attributes are generated on the multi-resolution surfaces. In order to assess the regularity and variability of the shape attributes for each ROI in the working memory network, we performed a set of statistical and computational analysis for ROIs within a population. The experimental results show that there exists deep-rooted regularity of cortical folding patterns for certain ROIs. Finally, we performed ROI prediction based on the extracted multi-scale folding attributes, and our results show that only certain ROIs with consistent folding patterns across individuals can be predicted accurately.

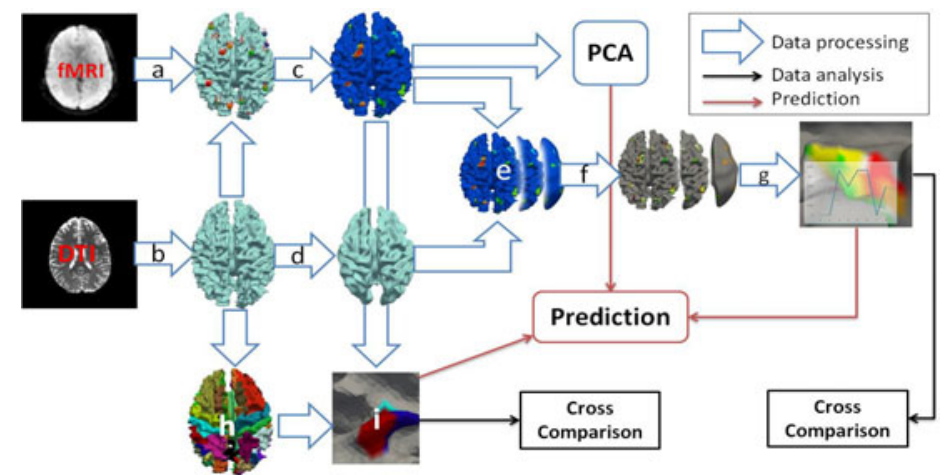

Fig. 1. The flowchart of our approaches. (a) Obtain working memory network ROIs from taskbased fMRI. (b) Reconstruct cortical surface from DTI data. (c) Map ROIs onto cortical surface. (d) Decompose cortical surface into multi-resolution surfaces. (e) Map ROIs on multiresolution surfaces. (f) Compute folding patterns. (g) Generate meso-scale attributes for each ROI. (h) Atlas labeling. (i) Generate global-scale attribute for each ROI.

\section{Methods}

\subsection{Overview}

The flowchart of the proposed algorithmic pipeline is outlined in Fig. 1. The folding pattern of human cerebral cortex is a multi-scale concept [9]. Hence, we extract shape features for each ROI base on both folding patterns of multi-resolution surfaces and MNI atlas labels, and then assess the regularity and variability of these features. The cortical surface reconstructed from DTI data [10] is decomposed into multiresolutions by the spherical wavelet algorithm approach [7]. The volumetric working 
memory ROIs were obtained from task-based fMRI, and then are mapped onto the reconstructed surface, as well as other decomposed multi-resolution surfaces. Then, the folding patterns of the ROIs are computed by the polynomial models [4], and the feature vectors of each ROI is then obtained and analyzed via the methods illustrated in Fig. 1 separately. Finally, ROI prediction based on these folding pattern features is performed and a PCA (principal component analysis) model is introduced to constrain the spatial relationship among ROIs.

\subsection{Data Acquisition and Pre-processing}

Seventeen healthy students were recruited to participate in this study. Each participant performed a modified version of the OSPAN task (3 block types: OSPAN, Arithmetic, and Baseline) while fMRI data was acquired. DTI scans were also acquired for each participant. FMRI and DTI scans were acquired on a 3T GE Signa scanner. Acquisition parameters are as follows: fMRI: 64x64 matrix, 4mm slice thickness, 220mm FOV, 30 slices, TR=1.5s, TE=25ms, ASSET=2; DTI: 128x128 matrix, 2mm slice thickness, 256mm FOV, 60 slices, TR=15100ms, ASSET=2, 3 B0 images, 30 optimized gradient directions, b-value $=1000$. Each participant's fMRI data was analyzed using FSL FEAT. Individual activation map reflecting the OSPAN (complex span) contrast was identified. Totally, 15 activated ROIs were recognized and applied for this study (Table 1). These ROIs were mapped on the cortical surface and on the corresponding decomposed surfaces, as shown in Fig. 2. The size of ROI is defined by the number of neighborhood vertex rings, which is set as 5 in this paper.

Table 1. The names and coresponding IDs of 15 recognized working memory ROIs

\begin{tabular}{|c|c|c|c|c|c|}
\hline \multirow[t]{2}{*}{ Region name } & \multicolumn{2}{|c|}{ Region ID } & \multirow[t]{2}{*}{ Region name } & \multicolumn{2}{|c|}{ Region ID } \\
\hline & Left & Right & & Left & Right \\
\hline Insula & 1 & 10 & Precuneus & 6 & 13 \\
\hline Medial Frontal Cortex & 2 & & Superior Frontal Gyrus & 7 & 14 \\
\hline Occipital Pole & 3 & & Inferior Parietal Lobule & 8 & 15 \\
\hline Paracingulate Gyrus & 4 & 12 & Dorsolateral Prefrontal Cortex & & 9 \\
\hline Precentral Gyrus & 5 & & Lateral Occipital Gyrus & & 11 \\
\hline
\end{tabular}

\subsection{Multi-scale Surface Decomposition}

In this paper, the over-complete spherical wavelets algorithm [7] is applied to decompose the original cortical surface reconstructed from DTI data into a cascade of 7 lower resolutions (Fig. 2). The lower the resolution is, the smoother and less convoluted the surface will be. In this spherical wavelet transform process, a set of wavelet coefficient samples on the subdivided icosahedron grid are obtained from the input shape described as a set of mesh vertices, which corresponds directly to the resolution level of the spherical wavelet transform. After the transformation, each vertex has a set of wavelet coefficients representing its position in lower resolutions. The total number of vertices and the indices of vertices do not change during this decomposition process [7]. Thus, the correspondence of a vertex remains in all resolutions, which is illustrated by the same ROI colors in Fig. 2. More details of the over-complete spherical wavelets algorithm are referred to [7]. 


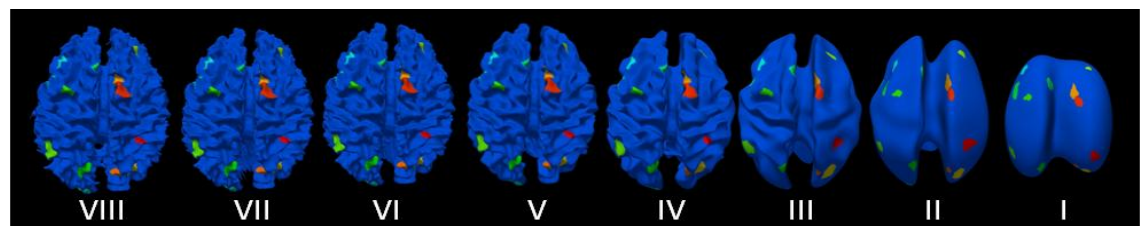

Fig. 2. An example of multi-resolution decomposition for a cortical surface. The ROIs are mapped onto all resolutions respectively encoded by certain colors.

\subsection{Folding Pattern Descriptors}

\subsubsection{Meso-Scale Attributes Based on Polynomial Folding Descriptors}

Based on the above surface decomposition, the folding pattern attributes of each ROI are extracted on multi-resolutions, respectively. To compute the folding pattern of a surface patch, a parametric folding descriptor using polynomials [4] is applied:

$$
Z=a X^{2}+b Y^{2}+c X^{3}+d Y^{3}
$$

where, $a$ and $b$ describe the mirror symmetric components of the patch along $\mathrm{x}$ axis and y axis respectively, while $c$ and $d$ represent the rotational symmetric components [4]. Then, a model-driven method similar to that in [4] is applied to classify the combination of the symmetric components into eight primitive patterns of peak, pit, ridge, valley, saddle ridge, saddle valley, flat, and inflection [8]. The attribute vector of ROI on each surface resolution is defined as the normalized histogram of eight patterns within ROI. Thus, there is an 8-dimensional attribute vector for each ROI on each resolution of surface representation. Hence, we have a 64-dimensional mesoscale folding pattern attribute vector for each ROI.

\subsubsection{Global-scale Folding Attribute Based on MNI Labels}

The HAMMER tool [12] was used to label the cortical surface into MNI labels. Then, the global-scale attribute vector of ROI based on MNI atlas labels is defined as the normalized histogram of MNI labels within each ROI as follows.

$$
V_{i}=\frac{\left(v_{i}^{h_{1}}, v_{i}^{h_{2}}, \cdots, v_{i}^{h_{n}}\right)}{\left|v_{i}^{h_{1}}, v_{i}^{h_{2}}, \cdots, v_{i}^{h_{n}}\right|}
$$

where $v_{i}^{h_{k}}$ is the number of vertices with MNI label $h_{k}$ within ROI $i$. The size of the vector is dependent on the combination of MNI label within an ROI.

\subsection{Folding Pattern Feature Analysis}

The cosine similarity has been widely applied as a measurement of similarity between feature vectors, and is adopted here. Denote $R_{m}^{p}$ as ROI \#m of subject $p$ and $A_{m}^{p}$ as the attribute vector of $R_{m}^{p}$, the average similarity $S_{m n}$ between 2 ROIs $m$ and $n$ is defined to measure the feature similarity for each ROI pair within a group as follows: 


$$
\begin{gathered}
S_{m n}=\frac{\sum_{p=1}^{N-1} \sum_{q=p+1}^{N} F\left(R_{m}^{p}, R_{n}^{q}\right)}{C_{N}^{2}} \\
F\left(R_{m}^{p}, R_{n}^{q}\right)=\frac{A_{m}^{p} \cdot A_{n}^{q}}{\left\|A_{m}^{p}\right\|\left\|A_{n}^{q}\right\|}
\end{gathered}
$$

where $N$ is the number of subjects.

Considering the length of global-scale attribute can be different, when comparing the similarity between them, the attribute vectors are normalized to the same length. Elements that represent certain MNI labels are viewed as the weights of attribute vector in certain direction. For those elements not shared between attributes, they are set to zero in order to unify the length of attribute vectors.

\subsection{ROI Prediction}

An ROI prediction framework is developed based on the cortical folding features described above. Before performing the ROI prediction, all of the subjects are aligned to the same template space (a randomly selected subject in the dataset) by using the linear image registration algorithm FSL FLIRT [13]. Then, the ROI prediction is formulated and solved by minimizing the energy function defined below:

$$
E=\lambda E_{\text {int }}+(1-\lambda) E_{\text {ext }}
$$

where internal term $E_{\text {int }}$ is the spatial constraint of ROIs' locations which regularizes the search of ROI in a certain space, and the external term $E_{\text {ext }}$ describes the distance between folding pattern feature vectors, and $\lambda$ trades off these two terms.

As there exists certain consistence in the spatial distribution pattern of ROIs, an ROI coordinate PCA model is introduced to constrain the spatial relationship among ROIs. $E_{\text {int }}$ is defined as the reconstruction error when projecting the candidate ROIs into the sub-space represented by the PCA model. The external energy for one candidate group is formulated as:

$$
E_{\text {ext }}=\frac{1}{n} \sum_{i=1}^{i=n}\left(\beta E_{\text {global }}^{i}+(1-\beta) E_{\text {meso }}^{i}\right)
$$

where $E_{\text {global }}^{i}, E_{m e s o}^{i}$ are the distances between global and meso scale folding pattern feature vectors of candidate ROI $\# i$ and those in the training set, and $\beta$ is the trade-off. The optimization is solved by search over the whole space.

\section{Results}

\subsection{Meso-scale Folding Pattern Analysis}

Quantitative assessments of the regularity and variability of the folding pattern attribute vectors for each ROI are summarized in Fig. 3. It is evident in Fig. 3(a) that the meso-scale attributes of certain ROIs including ROI \#1, \#2, and \#7, are less 
variable in that the first principal components can account over $85 \%$ of the variances. However, the attributes of other ROIs, including ROI \#6, have much more variability and the first principal components can only account for $68 \%$ of the variance. These results demonstrate that there is deep-rooted regularity of cortical folding patterns for specific working memory ROIs such as ROI \#1 and \#7 across subjects, and the folding pattern attributes could be potentially used as predictors of functional ROIs.

The similarities between attribute vectors of each pair of ROIs across 17 subjects were calculated in different resolution using the methods in section 2.5 and shown in Fig. 3(b). It can be seen from Fig. 3(b) that, in the first and second resolutions of surface, the 'flat' folding pattern dominates over others and the attribute vectors are almost the same in these two resolutions. However, starting with the third resolution, the attribute vectors are much more distinctive among ROIs which indicates that the distinctiveness of folding pattern attributes of ROIs is dependent on the resolutions of surface representations. If we look at the diagonals in the matrices in Fig. 3(b), some ROIs such as ROI \#2, \#3, \#7, and \#13, have higher similarity, while others such as ROI \#5 and \#11 have lower similarity. This result partly reflects the similar conclusion made by PCA analysis. An interesting finding in Fig. 3(b) is that ever since the fourth resolution, 4 dark lines can be obviously observed in the matrices, which are highlighted by blue arrow in Fig. 3(b.VIII). As dark represents low similarity, it means that the attribute vectors of ROI \#1 and ROI \#10 are very different from those of other ROIs and share unique patterns. An intriguing fact is that these two ROIs are left and right insular respectively. We used a classifier based on the support vector machine (SVM) [11] to differentiate these two ROIs from the rest by the folding attributes. The classification test was performed in a leave-one-out fashion. We obtained a relatively high true positive rate of $85.29 \%$ in test and a low false negative rate of $2.26 \%$. This promising result suggests that folding patterns can characterize certain brain ROIs (e.g., insular) and can provide distinctive morphological signatures for ROI recognition.

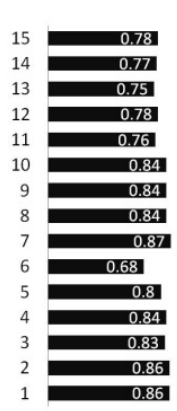

(a)
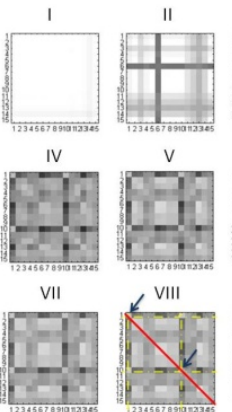

(b)
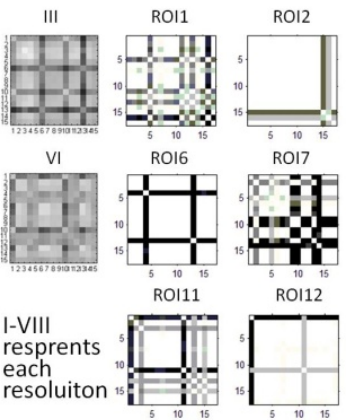

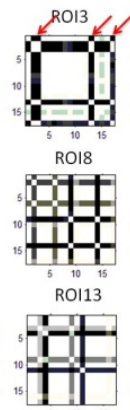

(c)

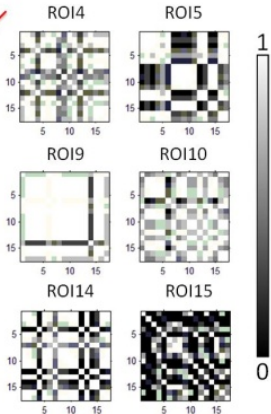

Fig. 3. (a): Histograms of percentages of variance that the first principal components can account for after applying PCA on meso-scale feature vectors of each ROI. (b): Average similarity $S_{m n}$ between meso-scale feature vectors of 15 ROIs on each resolution. Each line represents an ROI. Color bar is on the right. (c): Similarity of global-scale ROI attributes between 17 subjects. Each line represents a single subject. 


\subsection{Global-Scale Folding Pattern Analysis}

The similarities of global-scale attribute vectors defined in Eq. (2) of each ROI across 17 subjects were calculated using the methods in section 2.5 . The similarity of attribute of each ROI is shown in Fig. 3(c). It can be seen from Fig. 3(c) that, some ROIs such as ROI \#2, \#9, and \#12, have higher similarities across subject while others such as ROI \#15, are more variable across subjects. It is interesting that the ROI \#2 and \#9 are again more consistent across subjects in terms of meso-scale folding pattern features, as shown in Fig. 3(a-b). This result further indicates that the level of regularity and variability of functional ROI folding patterns is very ROI-specific.

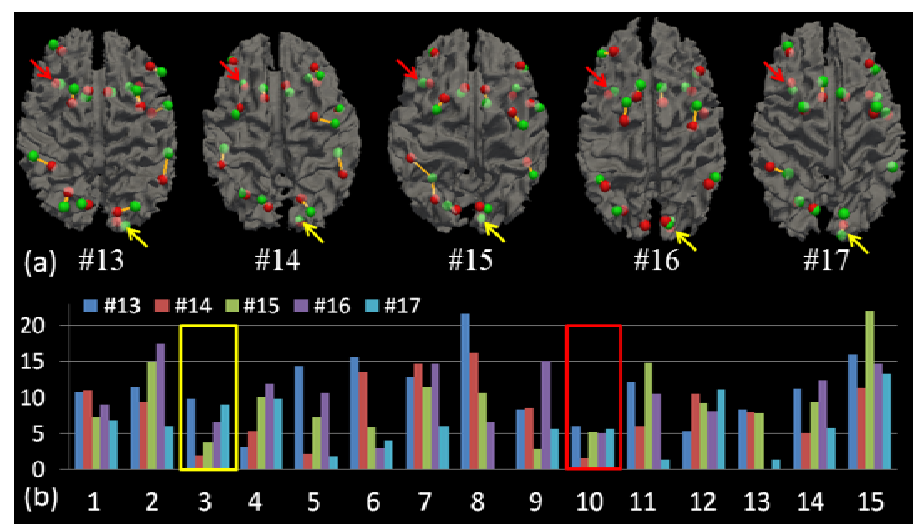

Fig. 4. (a) Prediction results. The center of benchmark ROIs and predicted ROIs are presented by green and red bubbles respectively and connected by yellow line for each pair. ROI \#3 and \#10 are highlighted by yellow and red arrows accordingly. (b) Euclidean distances between 15 predicted ROIs and benchmark ROIs for 5 subjects. The horizontal axis indexes ROI. ROI \#10 is highlighted with red for its good prediction result, in which the average error is $4.7 \mathrm{~mm}$.

\subsection{ROI Prediction}

We used 12 subjects from \#1-\#12 as training dataset to obtain the prior model of ROIs. Then, the prediction framework described in Section 2.6 was performed on the rest of 5 subjects (Fig. 4(a)). The prediction result was measured by Euclidean distance between predicted ROI and the benchmark ROI provided by task-based fMRI, as shown in Fig. 4(b). Among all of these 15 ROIs, the prediction accuracy is the best for ROI \#10 (average prediction error: $4.7 \mathrm{~mm}$ ), which significantly improved the initialized ROI positions by linear registration (average initialization error: $5.4 \mathrm{~mm}$ ). This can be explained by the relatively consistent meso-scale and global-scale folding patterns for ROI \#10 across subjects. Though the prediction results of ROI \#3 are better for subjects from \#14 - \#16, but worse for subjects \#13 and \#17. This is partially caused by the uniqueness of global scale features of these subjects, as highlighted by red arrows in Fig. 3(b). This result suggests that stratification of ROIs and subjects into sub-groups based on their folding patterns 
could facilitate ROI prediction within homogeneous subgroups and potentially improve ROI prediction accuracy. For other ROIs, our methods can not significantly improve the initialized ROI positions in that the folding pattern features of these ROIs are not consistent in either meso or global scale across individuals, as shown in Fig. 3.

It should be noted that although our ROI prediction results in Fig. 4 are preliminary, it suggests the possibility of using folding patterns to predict certain functional ROIs without the availability of task-based fMRI data. In many clinical applications in which there is no task-based fMRI data available, it would be very helpful to accurately predict certain functional ROIs based only on the folding pattern features that can be extracted from widely available structural MRI data.

\section{Conclusion}

We presented a novel framework to assess folding patterns of ROIs that are identified by working memory task-based fMRI. The regularity and variability of these attributes have been assessed within and across ROIs, as well as within and across subjects. The major conclusions of our work are as follows. (1) There are deep-rooted regularities of cortical folding patterns for specific working memory ROIs, e.g., insular, across subjects. However, the intrinsic relationship between the regularity of cortical folding patterns and brain connectivity and function remains to be elucidated in the future. (2) Folding pattern attributes, if extracted and applied in a proper way, could be very useful for the characterization, recognition and prediction of brain ROIs, e.g., ROI \#10 of insular in the working memory network in Fig. 4. In the absence of fMRI data, this ROI prediction capability based on widely available MRI data could be helpful in localizing functional brain regions in clinical applications.

\section{References}

1. Van Essen, D.C.: A tension-based theory of morphogenesis and compact wiring in the central nervous system. Nature 385(6614), 313-318 (1997)

2. Sporns, O., Chialvo, D.R., Kaiser, M., Hilgetag, C.C.: Organization, development and function of complex brain networks. Trends Cogn. Sci. 8(9), 418-425 (2004)

3. Passingham, R.E., Stephan, K.E., Kötter, R.: The anatomical basis of functional localization in the cortex. Nat. Rev. Neurosci. 3(8), 606-616 (2002)

4. Zhang, T., Guo, L., Li, G., Nie, J., Liu, T.: Parametric representation of cortical surface folding based on polynomials. In: Yang, G.-Z., Hawkes, D., Rueckert, D., Noble, A., Taylor, C. (eds.) MICCAI 2009. LNCS, vol. 5762, pp. 184-191. Springer, Heidelberg (2009)

5. Zilles, K., Armstrong, E., Schleicher, A., Kretschmann, H.J.: The human pattern of gyrification in the cerebral cortex. Anat. Embryol. (Berl.) 179, 173-179 (1988)

6. Yu, P., Yeo, B.T., Grant, P.E., Fischl, B., Golland, P.: Cortical Folding Development Study based on Over-Complete Spherical Wavelets. In: IEEE Workshop on MMBIA, pp. 1-8 (2007)

7. Yeo, B.T., Yu, P., Grant, P.E., Fischl, B., Golland, P.: Shape analysis with overcomplete spherical wavelets. In: Metaxas, D., Axel, L., Fichtinger, G., Székely, G. (eds.) MICCAI 2008, Part I. LNCS, vol. 5241, pp. 468-476. Springer, Heidelberg (2008) 
8. Besl, P.J., Jain, R.C.: Segmentation Through Variable-Order Surface Fitting. IEEE Transactions on Pattern Analysis and Machine Intelligence 10, 167-192 (1988)

9. Li, K., Guo, L., Li, G., Nie, J., Faraco, C., Cui, G., Zhao, Q., Miller, L.S., Liu, T.: Gyral folding pattern analysis via surface profiling. NeuroImage 52(4), 1202-1214 (2010)

10. Liu, T., Li, H., Wong, K., Tarokh, A., Guo, L., Wong, S.T.: Brain tissue segmentation based on DTI data. NeuroImage 38, 114-123 (2007)

11. Chang, C.-C., Lin, C.-J.: LIBSVM: a library for support vector machines. Software (2001), http://www.csie.ntu.edu.tw/cjlin/libsvm

12. Shen, D., Davatzikos, C.: HAMMER: hierarchical attribute matching mechanism for elastic registration. IEEE Trans. Med. Imaging 21(11), 1421-1439 (2002)

13. FMRIB Software Library, http: //www. fmrib.ox.ac.uk/fsl/index.html 